\title{
Neuro Fuzzy Model for Human Face Expression Recognition
}

\author{
Mr. Mayur S. Burange ${ }^{1}$, Prof. S. V. Dhopte ${ }^{2}$ \\ ${ }^{I}$ M.E.(Scholar), PRMI Engineering \& Technology,Badnera $(M H)$ \\ ${ }^{2}$ Associate Prof, PRMI Engineering \& Technology,Badnera $(\mathrm{MH})$
}

\begin{abstract}
This paper present an approach to recognize human face expression and emotions based on some fuzzy pattern rules. Facial features for this specially eye and lips are extracted an approximated into curves which represents the relationship between the motion of features and change of expression. This paper focuses the concepts like face detections, skin color segmentation, face features extractions and approximation and fuzzy rules formation. Conclusion based on fuzzy patterns never been accurate but still our intension is to put more accurate results.
\end{abstract}

Key words -- Face Detection, Skin Color Segmentation, Face Futures, Curve Formation and Approximation, Fuzzy Patterns.

\section{Introduction:}

Facial expression analysis has been attracted considerable attention in the advancement of human machine interface since it provides natural and efficient way to communicate between humans [2]. Some application area related to face and its expression includes personal identification and access control, video phone and teleconferencing, forensic application, human computer application [5].

Most of the facial expression recognition methods reported to date or focus on expression category like happy, sad, fear, anger etc. For description of detail face facial expression, Face Action Coding System (FACS) was design by Ekman[8]. In FACS motion of muscles are divided into 44 action units and facial expression are described by their combination. Synthesizing a facial image in model based image coding and in MPEG-4 FAPs has important clues in FACS. Using MPEG-4 FAPs, different 3D face models can be animated. Moreover, MPEG-4 high level expression FAP allows animating various facial expression intensities. However, the inverse problem of extracting MPEG-4 low and high level FAPs from real images is much more problematic due to the fact that the face is a highly deformable object [1].

\section{Literature Review}

Designer of FACS, Ekman himself as pointed out some of these action units as unnatural type facial movements. Detecting a unit set of action units for specific expression is not guaranteed. One promising approach for recognizing up to facial expressions intensities is to consider whole facial image as single pattern [4]. Kimura and his colleagues have reported a method to construct emotional space using $2 \mathrm{D}$ elastic net model and K-L expansions for real images [7]. Their model is user independent and gives some unsuccessful results for unknown persons. Later Ohba proposed facial expression space employing principle component analysis which is person dependant [9].

This project consists of following phases:

\section{Proposed Method:}

3.1. Face detection based on skin color

3.2. Face extraction and enhancement

3.3. Face features extraction

3.4. Curve formation using Bezier curve.

3.5. Fuzzy Patterns

3.6. Experiment Results

\subsection{Face Detection Based on Skin Color:}

Skin color plays a vital role in differentiating human and non-human faces. From the study it is observe that skin color pixels have a decimal value in the range of 120 to 140. In this project, we used a trial and error method to locate skin color and non skin color pixels. But many of the times, system fails to detect whether an image contains human face or not (i.e. for those images where there is a skin color background).an image is segmented into skin color and non-skin color pixels with the equations 
$120 \leq|P x y| \leq 140---------e q .3 .1 .1$

$$
\text { where Pxy = pixel at position } x y
$$

The skin pixels values are set to 1(i.e. \#FFFF) and non skin pixels are set to 0(i.e. 0000). The pixels are collected and set as per equation

If

$\lim _{i \rightarrow 1} n\left(\int_{1}^{3} 120 \leq|P x y| \leq 140\right)=1-------$ eq3.1.2

Else

$\lim _{i \rightarrow 1} n\left(\int_{1}^{3} 140 \leq|P x y| \leq 120\right)=0$-------eq 3.1 .3

where $n=$ total number of pixels of input image

The resultant image becomes as

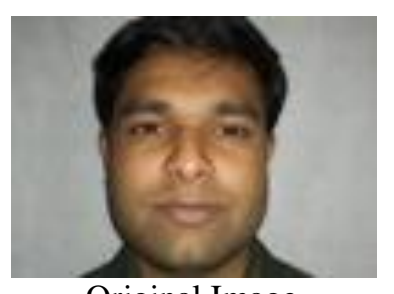

Original Image

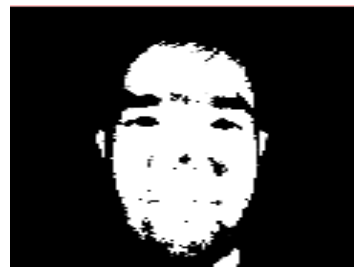

Skin and non-skin pixels

Fig. 3.1.1 (Phase I)

\subsection{Face Extraction and Enhancement}

Literature review point out that, FACS system technique is based on face features extractions like eye, nose, mouth, etc. In this project, we minimize the number of features (i.e. only eyes and mouth) but given the more weightage for fuzzy rules formations from these extracted features. Face extractions consist of following steps

- Let $\mathrm{W}$ and $\mathrm{H}$ are the width and height of skin and non-pixel image as shown in fig 3.1.1

- Read the pixel at position $(0, \mathrm{H} / 2)$ which is a middle of i.e. left side of image.

- Travers a distance $D_{1}=W / 6$ in horizontal direction to get the start boundary pixel of skin region.

- Travers a distance $\mathrm{D}_{2}=\mathrm{H} / 6$ from a pixel position $(\mathrm{W} / 6, \mathrm{H} / 2)$ in upward directions. Same may do in downward direction and locate the points $\mathrm{X}_{1}, \mathrm{X}_{2}$.

- Travers a distance $\mathrm{D}_{3}=\mathrm{W} / 3$ from the point $\mathrm{X}_{1}$ and locate the point $\mathrm{X}_{3}$. Same do from the point $\mathrm{x}_{2}$ and locate the point $\mathrm{X}_{4}$.

- Crop the square image as shown.
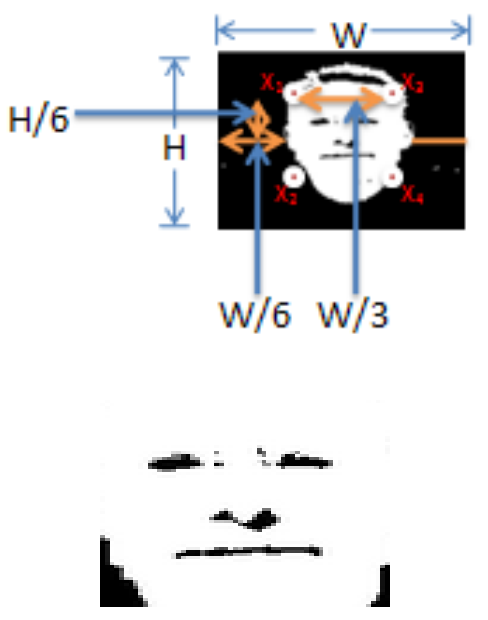

www.iosrjournals.org 
Fig 3.2.2 Face recognition

After face extraction white region pixels (i.e. skin pixels) are filled with skin color. A resultant image with skin color and after enhancement becomes as

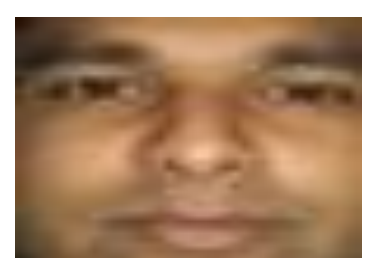

Fig 3.2.3 image with skin color and after dimension enhancement

\subsection{Face Features Extraction}

Human face is made up of eyes; nose, mouth and chine etc. there are differences in shape, size, and structure of these organs. So the faces are differs in thousands way. One of the common methods for face expression recognition is to extract the shape of eyes and mouth and then distinguish the faces by the distance and scale of these organs. The face feature extractions consist of following steps

- Let $\mathrm{W}$ and $\mathrm{H}$ are width and height of an image shown in Fig 3.2.3

- Mark pixel $\mathrm{P}_{\mathrm{i}}(\mathrm{W} / 2, \mathrm{H} / 2)$ as centre of image.

- Travers a distance $\mathrm{H} / 8$ from the pixel $\mathrm{P}_{\mathrm{i}}$ towards upward and mark a point $\mathrm{K}_{1}$.

- Travers a distance $\mathrm{W} / 3$ from the point $\mathrm{K}_{1}$ towards leftward and mark a point $\mathrm{K}_{2}$.

- Travers a distance $\mathrm{H} / 10$ towards downward from the point $\mathrm{K}_{2}$ and mark a point $\mathrm{K}_{3}$.

- Travers a distance $\mathrm{W} / 4$ from the point $\mathrm{K}_{3}$ towards right and mark the point $\mathrm{K}_{4}$.

- Travers a distance $\mathrm{H} / 10$ from the point $\mathrm{K}_{4}$ toward up and mark the point $\mathrm{K}_{5}$.

- Same steps are repeated for extracting the right eye and mark the point $\mathrm{N}_{2}, \mathrm{~N}_{3}, \mathrm{~N}_{4}$, and N5.

- Travers a distance $\mathrm{H} / 8$ from the point $\mathrm{P}_{\mathrm{i}}$ towards downward and mark the point $\mathrm{M}_{1}$.

- Travers a distance W/6 towards left and right from the point $\mathrm{M}_{1}$ and marks the point $\mathrm{M}_{2}$ and $\mathrm{M}_{3}$.

- Start with the point $\mathrm{M}_{2}$ traverse a distance $\mathrm{H} / 10$ towards downward and mark the point $\mathrm{M}_{4}$.

- Travers a distance W/6 from the point $\mathrm{M}_{4}$ towards right and mark the point $\mathrm{M}_{5}$. Same may do from point $\mathrm{M}_{5}$ and mark the point $\mathrm{M}_{6}$.

- Travers the distance $\mathrm{H} / 10$ from $\mathrm{M}_{6}$ towards up that meets to the point $\mathrm{M}_{3}$.

- See the below image.

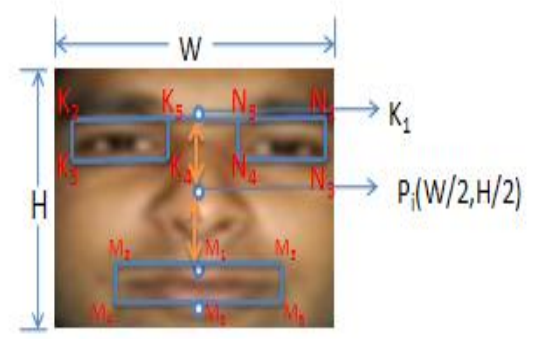

Fig 3.3.1 Feature Extraction

- $\quad$ Dist $\left|\mathrm{P}_{\mathrm{i}}-\mathrm{K}_{1}\right|=\mathrm{H} / 8$

- $\quad$ Dist $\left|\mathrm{K}_{1}-\mathrm{K}_{2}\right|=$ Dist $\left|\mathrm{M}_{1}-\mathrm{M}_{2}\right|=$ Dist $\left|\mathrm{M}_{1}-\mathrm{M}_{3}\right|=$ $\operatorname{Dist}\left|M_{4}-M_{5}\right|=\operatorname{Dist}\left|M_{5}-M_{6}\right|=W / 3$

- Dist $\left|\mathrm{K}_{2}-\mathrm{K}_{3}\right|=$ Dist $\left|\mathrm{K}_{4}-\mathrm{K}_{5}\right|=$ Dist $\left|\mathrm{N}_{2}-\mathrm{N}_{3}\right|=$

Dist $\left|N_{4}-N_{5}\right|=\operatorname{Dist}\left|M_{2}-M_{4}\right|=\operatorname{Dist}\left|M_{1}-M_{5}\right|=$

Dist $\left|M_{3}-M_{6}\right|=H / 10$

- Dist $\left|\mathrm{K}_{3}-\mathrm{K}_{4}\right|=$ Dist $\left|\mathrm{K}_{5}-\mathrm{K}_{2}\right|=$ Dist $\left|\mathrm{N}_{3}-\mathrm{N}_{4}\right|=$ www.iosrjournals.org 


$$
\text { Dist }\left|\mathrm{N}_{5}-\mathrm{N}_{2}\right|=\mathrm{W} / 4
$$

\subsection{Curve formation using Bezier curve}

Eyes and mouth as shown in fig 3.3.1 are located and extracted. Bezier curve formed from this eyes and mouth as per the equation

$Q(t)=\sum_{i=0}^{n} P_{i} B_{i, n}(t)$

Where each term in the sum is the product of blending function $B_{i, n}(t)$ and the control point $P_{i .}$. The $B_{i, n}(t)$ is called as Bernstein polynomials and are defined by

$B_{i, n}(t)=C_{i}^{n} t^{i}(1-t)^{n-i}$ .eq. 3.4.2

Where $C_{i}^{n}$ is the binomial co-efficient given by:

$C_{i}^{n}=\frac{n !}{i !(n-i)}$.

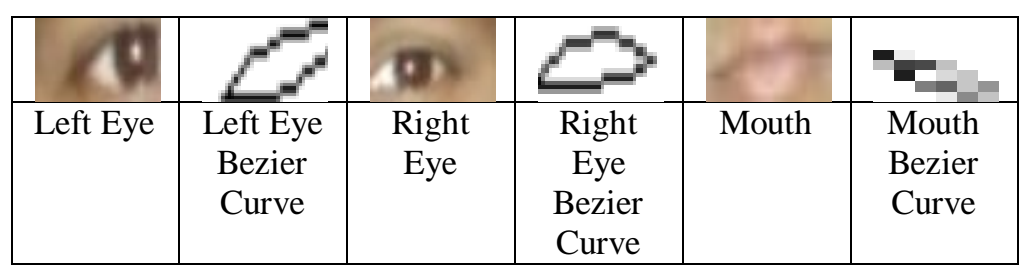

Fig 3.4.1 Bezier Curve

Once the Bezier curve formed features points are located as shown in below image.

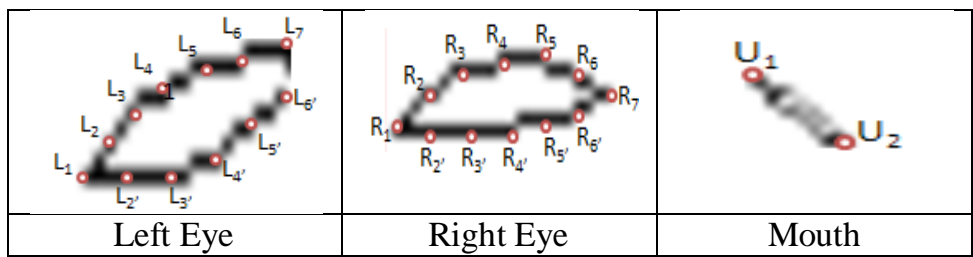

Fig 3.4.2 Feature Point Location

The feature point distance for left and right eye is measured with

$$
\begin{array}{r}
\mathrm{Z}=\int_{i}^{n} \sum_{i=2}^{6}\left(e^{H_{i}} \sin w_{i}-e^{H_{i^{\prime}}} \cos w_{i}\right) \ldots \ldots . . \text { eq. } 3.4 .4 \\
\text { where } Z=\text { feature point distance } \\
n=\text { number of feature points }
\end{array}
$$

For left eye $\mathrm{H}_{\mathrm{i}}=\mathrm{L}_{\mathrm{i}}$ and for right eye $\mathrm{H}_{\mathrm{i}}=\mathrm{R}_{\mathrm{i}}$.

The feature point distance for mouth is measured with

$Z^{\prime}=\int_{i}^{n} \sum_{i=1}^{2}\left(e^{H_{i}} \sin w_{i} / 2-e^{H_{i^{\prime}}} \cos w_{i} / 2\right) \quad \ldots$ eq.3.4.5

An expression id generated from an average of $Z$ and $Z$ ' as below.

$\mathrm{id}=\left(\mathrm{Z}+\mathrm{Z}^{\prime}\right) / 2$ ..eq.3.4.6 


\subsection{Fuzzy Patterns}

It is found that expression recognition from the still image never gives a correct output. A one expression id may also false into more than one expression domain. This project forms some fuzzy patterns for expressions. See the set theory diagram below

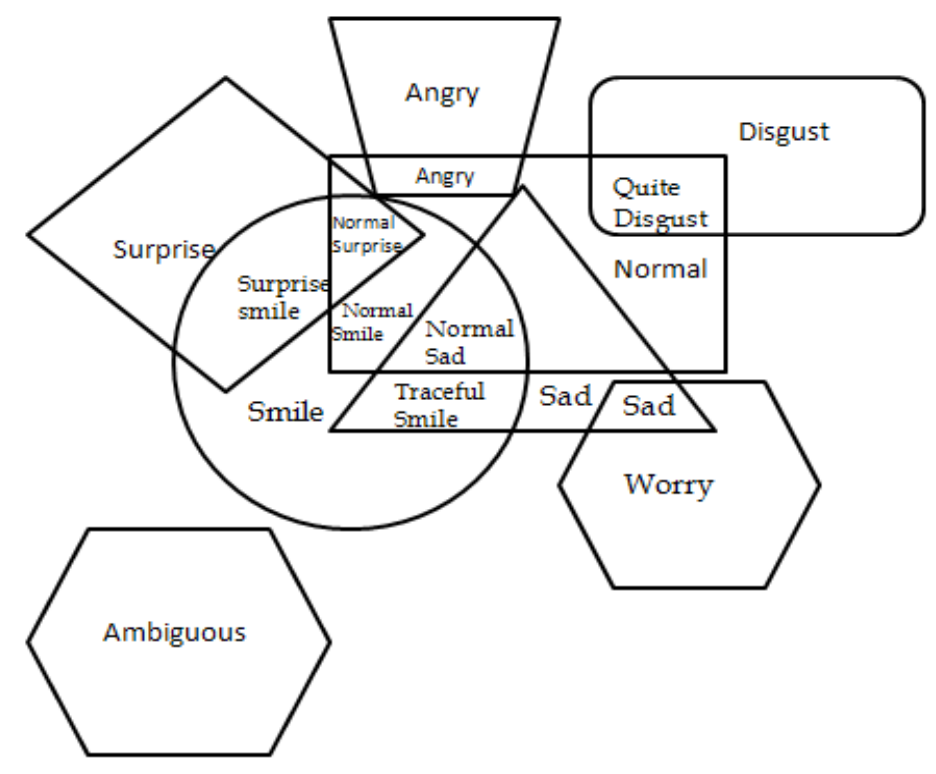

Fig 3.5.1 Fuzzy Expression Patterns

\subsection{Experiment Results}

\begin{tabular}{|c|c|c|c|c|c|c|}
\hline $\begin{array}{l}\text { Sr. } \\
\text { No. }\end{array}$ & Image Format & Dimension & $\begin{array}{c}\text { No. Images } \\
\text { tested }\end{array}$ & $\begin{array}{c}\text { Face } \\
\text { recognition }\end{array}$ & $\begin{array}{c}\text { Face } \\
\text { Extraction }\end{array}$ & $\begin{array}{l}\text { Expression } \\
\text { Accuracy }\end{array}$ \\
\hline 1 & JPEG & $50 * 50$ & 50 & $x$ & $x$ & $x$ \\
\hline 2 & JPEG & $100 * 100$ & 50 & $\checkmark$ & $x$ & $90 \%$ \\
\hline 3 & JPEG & $200 * 200$ & 50 & $\checkmark$ & $\checkmark$ & $\checkmark$ \\
\hline 4 & JPEG & $500 * 500$ & 50 & $\checkmark$ & v & $\checkmark$ \\
\hline 5 & PNG & $50 * 50$ & 50 & $x$ & $\mathrm{x}$ & $\mathrm{x}$ \\
\hline 6 & PNG & $100 * 100$ & 50 & $v$ & $x$ & $88 \%$ \\
\hline 7 & PNG & $200 * 200$ & 50 & v & v & $v$ \\
\hline 8 & PNG & $500 * 500$ & 50 & v & $v$ & $v$ \\
\hline 9 & BMP & $50 * 50$ & 50 & $x$ & $\mathrm{x}$ & $\mathrm{x}$ \\
\hline 10 & BMP & $100 * 100$ & 50 & v & $x$ & $88 \%$ \\
\hline
\end{tabular}

\begin{tabular}{|c|c|c|c|c|c|c|}
\hline 11 & BMP & $200 * 200$ & 50 & $\checkmark$ & V & $\checkmark$ \\
\hline 12 & BMP & $500 * 500$ & 50 & $v$ & $v$ & $\checkmark$ \\
\hline 13 & TIFF & $50 * 50$ & 50 & $x$ & $x$ & $x$ \\
\hline 14 & TIFF & $100 * 100$ & 50 & $v$ & $x$ & $83 \%$ \\
\hline 15 & TIFF & $200 * 200$ & 50 & $\checkmark$ & 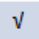 & $\checkmark$ \\
\hline 16 & TIFF & $500 * 500$ & 50 & $\checkmark$ & $v$ & $\checkmark$ \\
\hline 17 & GIF & $50 * 50$ & 50 & $x$ & $x$ & $x$ \\
\hline 18 & GIF & $100 * 100$ & 50 & $\checkmark$ & $x$ & $80 \%$ \\
\hline 19 & GIF & $200 * 200$ & 50 & $\checkmark$ & $\checkmark$ & $\checkmark$ \\
\hline 20 & GIF & $500 * 500$ & 50 & $\checkmark$ & $v$ & $v$ \\
\hline
\end{tabular}

Table 3.6.1 Result Analysis 


\section{Conclusion}

This paper proposes a new approach for recognizing the category of facial expression an estimating the degree of continuous facial expression change from time sequential images. This approach is based on personal independent average facial expression model.

\section{Future Work}

It is found that, current system fails to recognize an expression of images containing skin color background and multiple faces in single image. A strong system is required to diagnose an expression of such image.

\section{References}

[1] Y. Yacoob and L.S. Davis, "Recognizing human facial expressions from long image sequences using optical flow", IEEE Trans. Pattern Analysis \& Machine Intelligence, Vol. 18, No 6, pp. 636-642, 1996.

[2] P. Ekman and W. Friesen, "Facial Action Coding System", Consulting Psychologists Press, 1977.

[3] K. Aizawa and T. S. Huang, "Model-based image coding: Advanced video coding techniques for very low bit-rate applications", Proc. IEEE, Vol. 83, No. 2, pp. 259-271, 1995.

[4] S. Kimura and M. Yachida, "Facial expression recognition and its degree estimation", Proc. Computer Vision and Pattern Recognition, pp. 295-300, 1997.

[5] K. Ohba, G. Clary, T. Tsukada, T. Kotoku, and K. Tanie, "Facial expression communication with FES", Proc. International Conference on Pattern Recognition, pp. 1376-1378, 1998.

[6] M.A. Bhuiyan and H. Hama, "Identification of Actors Drawn in Ukiyoe Pictures", Pattern Recognition, Vol. 35, No. 1, pp. 93-102, 2002.

[7] M. B. Hmid and Y.B. Jemaa, Fuzzy Classification, Image Segmentation and Shape Analysis for Human Face Detection. Proc. Of ICSP, vol. 4, 2006.

[8] M. Wang, Y. Iwai, M. Yachida, "Expression Recognition from Time-Sequential Facial Images by use of Expression Change Model", Proc. Third IEEE International Conference on Automatic Face and Gesture Recognition, pp. 324 - 329, 1998.

[9] M. I. Khan and M. A. Bhuiyan, "Facial Expression recognition for Human-Machine Interface”, ICCIT, 2006. 